\title{
Improved strategy for computation of population mean under double stratified sampling framework
}

\author{
S. M. ZEESHAN AND G. K. VISHWAKARMA
}

\begin{abstract}
The article contains a new technique to estimate the mean of the variate of the interest of the finite population with the help of two auxiliary variates. The technique complies well with the stratified population in which each strata proportion is predicted by taking an initial sample called the first phase sample. When the first phase sample is taken, a second sample is taken from the first sample which is called the second phase sample which is used to estimate the mean of the variate of the interest. In our study, we have considered the population which has two correlated auxiliary variates that pass almost through the origin. In such a situation ratio estimation technique and product estimation technique that provides improved estimates of the mean of the variate of the interest. Our technique considers a ratio-product type exponential estimator of which we have established efficiency theoretically as well as empirically.
\end{abstract}

Mathematics Subject Classification 2000: 62D05

Keywords: Auxiliary variate, Study variate, Double stratified sampling scheme, Bias, Mean square error.

\section{INTRODUCTION}

In sample survey, surveyors use the auxiliary variates in order to increase the accuracy in the estimation of population parameters when the associated variates are readily and inexpensively available to the entire population. Many times the associated variates are not readily available, in which case, a larger sample is taken provided the collection of information on auxiliary variate is cheap, easy and economical. In many surveys, gathering information on variate of the interest is quite costly and gaining information on auxiliary variates are cheap, easy and economical or auxiliary variate are easily available. If the variate of the interest and auxiliary variates are highly correlated then the efficiency of the estimators may be increased by using auxiliary variate. But this will benefit only if the attainment in precision that is achieved by using the sample auxiliary variate weigh up the loss in precision that has been incurred because of the reduction in the size of the sample for computing mean of the variate of the interest. Surveyors also use auxiliary variates to estimate the population heterogeneity when there is absence of information about 
heterogeneity. Thus, in order to estimate the population mean of different levels of study, our population study is divided into different levels and sample observations have been prepared from each level using the double sampling method.

This article has drawn inspiration from many of the articles like Chand, 1975 and its chain type estimator for the double sampling, Bahl and Tuteja, 1991 product and ratio type exponential estimators, Upadhyay and Singh, 1999 noteworthily contributed in this area and motivated Kadilar and Cingi, 2003, Kadilar and Cingi, 2005, Shabbir and Gupta, 2005. Singh and Vishwakarma, 2007 evolved the method further. Samiuddin and Hanif, 2007, Singh et. al., 2011, Sanaulla et. al., 2014 and Vishwakarma and Singh, 2015 have used two auxiliary variates for double stratified sampling method. All in all, inspired by their work, a need was felt to propose a new methodology in this area, and the results are discussed in a subsequent section.

\section{DOUBLE STRATIFIED RANDOM SAMPLING}

Let there be a finite population, $U_{j}(j=1,2,3, \ldots, N)$, of size $N$ that can be divided into strata. Let the variates that can be measured with each unit be a target variate $y$ and $x$ and $z$ be the associated variates that information is either fully available or if not then it can be measured easily and economically. Let $L$ be the number of strata that can be formed from the population. Let $N_{h}(h=1,2,3, \ldots, L)$ be size of $h^{t h}$ - stratum such that $\quad \sum_{a}^{b} N_{h}=N \quad W_{h}=N_{h} / N$ and Let $\left(\bar{y}_{h}=\sum_{i=1}^{n_{h}} y_{h i} / n_{h}, \bar{x}_{h}=\sum_{i=1}^{n_{h}} x_{h i} / n_{h}, \bar{z}_{h}=\sum_{i=1}^{n_{h}} z_{h i} / n_{h}\right)$ be the unbiased estimators of the variate of the interest and the associated auxiliary variates of the population mean $\left(\bar{Y}_{h}=\sum_{i=1}^{N_{h}} y_{h i} / N_{h}, \bar{X}_{h}=\sum_{i=1}^{N_{h}} x_{h i} / N_{h}, \bar{Z}_{h}=\sum_{i=1}^{N_{h}} z_{h i} / N_{h}\right)$, based on $n_{h}$ observations. When information on $\bar{X}_{h}$ is unknown Double Stratified Sampling method comes to rescue by using first phase sample to note down value on auxiliary variates. The procedures for double stratified sampling method is to select a sample of size $n_{h}^{\prime}$ from the $h^{\text {th }}$ stratum using simple random sampling without replacement (SRSWOR) where $\sum_{h=1}^{L} n_{h}^{\prime}=n^{\prime}$ and note down the value on the observed auxiliary variate(s) for these units. This will be called a stratified first phase sample. Now, for noting down the 
information on variate of the interest, another sample of size $n_{h}^{\prime}\left(n_{h}<n_{h}^{\prime}\right)$ is drawn from the each stratum using SRSWOR such that $\sum_{h=1}^{L} n_{h}=n$. This will be called second phase sample.

Now, for the given conditions below:

$$
\begin{aligned}
& \bar{Y}=\sum_{h=1}^{L} W_{h} \bar{Y}_{h}, \bar{y}_{h}=\bar{Y}_{h}\left(1+e_{0 h}\right), e_{0}=\sum_{h=1}^{L} W_{h} \bar{Y}_{h} e_{0 h} / \bar{Y}, \\
& \bar{X}=\sum_{h=1}^{L} W_{h} \bar{X}_{h}, \bar{x}_{h}^{\prime}=\bar{X}_{h}\left(1+e_{1 h}^{\prime}\right), e_{1}=\sum_{h=1}^{L} W_{h} \bar{X}_{h} e_{1 h}^{\prime} / \bar{X}, \\
& \bar{X}_{h}=\bar{X}_{h}\left(1+e_{1 h}\right), e_{1}=\sum_{h=1}^{L} W_{h} \bar{X}_{h} e_{1 h} / \bar{X}, \\
& \bar{Z}=\sum_{h=1}^{L} W_{h} \bar{Z}_{h}, \bar{z}_{h}^{\prime}=\bar{Z}_{h}\left(1+e_{2 h}^{\prime}\right), e_{2}=\sum_{h=1}^{L} W_{h} \bar{Z}_{h} e_{2 h}^{\prime} / \bar{Z}, \\
& \bar{z}_{h}=\bar{Z}_{h}\left(1+e_{2 h}\right), e_{2}=\sum_{h=1}^{L} W_{h} \bar{Z}_{h} e_{2 h} / \bar{Z},
\end{aligned}
$$

The expectations are defined as,

$$
\left.\begin{array}{l}
E\left(e_{0}\right)=E\left(e_{1}\right)=E\left(e_{1}^{\prime}\right)=E\left(e_{2}\right)=E\left(e_{2}^{\prime}\right)=0 \\
E\left(e_{0}^{2}\right)=\frac{1}{\bar{Y}^{2}} \sum_{h=1}^{L} W_{h}^{2} f_{1 h} S_{y h}^{2}=V_{200} \\
E\left(e_{1}^{2}\right)=\frac{1}{\bar{X}^{2}} \sum_{h=1}^{L} W_{h}^{2} f_{1 h} S_{x h}^{2}=V_{020} \\
E\left(e_{1}^{\prime 2}\right)=\frac{1}{\bar{X}^{2}} \sum_{h=1}^{L} W_{h}^{2} f_{2 h} S_{x h}^{2}=V_{020}^{\prime} \\
E\left(e_{2}^{2}\right)=\frac{1}{\bar{Z}^{2}} \sum_{h=1}^{L} W_{h}^{2} f_{1 h} S_{z h}^{2}=V_{002} \\
E\left(e_{2}^{\prime 2}\right)=\frac{1}{\bar{Z}^{2}} \sum_{h=1}^{L} W_{h}^{2} f_{2 h} S_{z h}^{2}=V_{002}^{\prime}
\end{array}\right\}
$$




$$
\left.\begin{array}{l}
E\left(e_{0} e_{1}^{\prime}\right)=\frac{1}{\overline{Y X}} \sum_{h=1}^{L} W_{h}^{2} f_{2 h} S_{y x h}=V_{110}^{\prime}, E\left(e_{0} e_{1}\right)=\frac{1}{\overline{Y X}} \sum_{h=1}^{L} W_{h}^{2} f_{1 h} S_{y x h}=V_{110}, \\
E\left(e_{0} e_{2}^{\prime}\right)=\frac{1}{\bar{X} \bar{Z}} \sum_{h=1}^{L} W_{h}^{2} f_{2 h} S_{y z h}=V_{101}^{\prime}, E\left(e_{0} e_{2}\right)=\frac{1}{\overline{Y Z}} \sum_{h=1}^{L} W_{h}^{2} f_{1 h} S_{y z h}=V_{101}, \\
E\left(e_{1}^{\prime} e_{1}\right)=\frac{1}{\bar{X}^{2}} \sum_{h=1}^{L} W_{h}^{2} f_{2 h} S_{x h}^{2}=V_{020}^{\prime}, E\left(e_{1}^{\prime} e_{2}^{\prime}\right)=\frac{1}{\bar{X} \bar{Z}} \sum_{h=1}^{L} W_{h}^{2} f_{2 h} S_{z x h}=V_{011}^{\prime}, \\
E\left(e_{1}^{\prime} e_{2}\right)=\frac{1}{\bar{X} \bar{Z}} \sum_{h=1}^{L} W_{h}^{2} f_{2 h} S_{z x h}=V_{011}^{\prime}, E\left(e_{1} e_{2}^{\prime}\right)=\frac{1}{\bar{X} \bar{Z}} \sum_{h=1}^{L} W_{h}^{2} f_{2 h} S_{z x h}=V_{011}^{\prime}, \\
E\left(e_{1} e_{2}\right)=\frac{1}{\bar{X} \bar{Z}} \sum_{h=1}^{L} W_{h}^{2} f_{1 h} S_{z x h}=V_{011}, E\left(e_{2} e_{2}\right)=\frac{1}{\bar{Z}{ }^{2}} \sum_{h=1}^{L} W_{h}^{2} f_{2 h} S_{z h}^{2}=V_{002}^{\prime}
\end{array}\right\}
$$

where $f_{1 h}=\left(\frac{1}{n_{h}}-\frac{1}{n_{h}^{\prime}}\right), f_{2 h}=\left(\frac{1}{n_{h}^{\prime}}-\frac{1}{N_{h}}\right) f_{3 h}=\left(f_{1 h}-f_{2 h}\right)$

$$
\begin{aligned}
& S_{h y}^{2}=\frac{1}{N_{h}-1} \sum_{i=1}^{N_{h}}\left(y_{h i}-\bar{Y}_{h}\right)^{2}, S_{h x}^{2}=\frac{1}{N_{h}-1} \sum_{i=1}^{N}\left(x_{h i}-\bar{X}_{h}\right)^{2}, \\
& S_{h z}^{2}=\frac{1}{N_{h}-1} \sum_{i=1}^{N_{h}}\left(z_{h i}-\bar{Z}_{h}\right)^{2}, S_{h y x}=\frac{1}{N_{h}-1} \sum_{i=1}^{N_{h}}\left(y_{h i}-\bar{Y}_{h}\right)\left(x_{h i}-\bar{X}_{h}\right), \\
& S_{h y z}=\frac{1}{N_{h}-1} \sum_{i=1}^{N_{h}}\left(y_{h i}-\bar{Y}_{h}\right)\left(z_{h i}-\bar{Z}_{h}\right), S_{h x z}=\frac{1}{N_{h}-1} \sum_{i=1}^{N_{h}}\left(x_{h i}-\bar{X}_{h}\right)\left(z_{h i}-\bar{Z}_{h}\right) .
\end{aligned}
$$

\section{SOME AVAILABLE ESTIMATOR}

Some of the popular estimators that are available in the literature are reproduced here. Usual unbiased estimator for population mean $\bar{Y}$ in case of double stratified random sampling, is given by

$$
\bar{y}_{(s t)}=\sum_{h=1}^{L} W_{h} \bar{y}_{h},
$$

where

$$
W_{h}=\frac{N_{h}}{N}
$$

The variance of the unbiased estimator is,

$$
\operatorname{Var}\left(\bar{y}_{(s t)}\right)=\bar{Y}^{2}\left(\frac{\sum_{h=1}^{L} W_{h}^{2} f_{1 h} S_{y h}^{2}}{\bar{Y}^{2}}\right)=\bar{Y}^{2} V_{200}
$$


The ratio and product estimators for double stratified random sampling is,

$$
\hat{\bar{Y}}_{R d}=\bar{y}_{s t} \frac{\bar{x}_{s t}^{\prime}}{\bar{x}_{s t}}=\sum_{h=1}^{L} W_{h} \bar{y}_{h}\left(\frac{\sum_{h=1}^{L} W_{h} \bar{x}_{h}^{\prime}}{\sum_{h=1}^{L} W_{h} \bar{x}_{h}}\right)
$$

and

$$
\hat{\bar{Y}}_{P d}=\bar{y}_{s t} \frac{\bar{x}_{s t}}{\bar{x}_{s t}^{\prime}}=\sum_{h=1}^{L} W_{h} \bar{y}_{h}\left(\frac{\sum_{h=1}^{L} W_{h} \bar{x}_{h}}{\sum_{h=1}^{L} W_{h} \bar{x}_{h}^{\prime}}\right)
$$

The mean square error of above estimators, for the first order approximation are,

$$
\operatorname{MSE}\left(\hat{\bar{Y}}_{R d}\right)=\bar{Y}^{2}\left(V_{200}+V_{020}-V_{020}^{\prime}-2\left(V_{110}-V_{110}^{\prime}\right)\right)
$$

and

$$
\operatorname{MSE}\left(\hat{\bar{Y}}_{P d}\right)=\bar{Y}^{2}\left(V_{200}+V_{020}-V_{020}^{\prime}+2\left(V_{110}-V_{110}^{\prime}\right)\right)
$$

Mohanty (1967) regression-cum-ratio and regression-cum-product type estimator for $\mathrm{Y}$ in two-phase stratified sampling, is given by

$$
y_{r e g}^{R}=\left[\bar{y}_{s t}+\beta_{1}\left(\bar{x}_{s t}^{\prime}-\bar{x}_{s t}\right)\right] \frac{\bar{Z}}{\bar{z}_{s t}^{\prime}}
$$

and

$$
y_{r e g}^{P}=\left[\bar{y}_{s t}+\beta_{2}\left(\bar{x}_{s t}^{\prime}-\bar{x}_{s t}\right)\right] \frac{\bar{z}_{s t}^{\prime}}{\bar{Z}}
$$

where $\beta_{1}$ and $\beta_{2}$ are constants.

The MSE of $y_{\text {reg }}^{R}$ and $y_{\text {reg }}^{P}$ with $\beta_{1}$ and $\beta_{2}$ i.e. $\beta_{1}=\beta_{2}=\frac{\bar{Y}\left(\bar{V}_{110}-\bar{V}_{110}^{\prime}\right)}{\bar{X}\left(V_{020}-V_{020}^{\prime}\right)}$ is given by

$$
\operatorname{MSE}\left(y_{\text {reg }}^{R}\right)=\bar{Y}^{2}\left[V_{200}+V_{020}^{\prime}-2 V_{101}^{\prime}-\frac{\left(V_{110}^{\prime}-V_{110}\right)^{2}}{V_{020}-V_{020}^{\prime}}\right]
$$

and

$$
\operatorname{MSE}\left(y_{\text {reg }}^{R}\right)=\bar{Y}^{2}\left[V_{200}+V_{020}^{\prime}+2 V_{101}^{\prime}-\frac{\left(V_{110}^{\prime}-V_{110}\right)^{2}}{V_{020}-V_{020}^{\prime}}\right]
$$


Sanauallah et. al. (2014) generalized exponential chain ratio type estimators for the stratified two-phase sampling method is,

$$
\hat{\bar{Y}}_{S}=\lambda \sum_{h=1}^{L} W_{h} \bar{y}_{h}\left[\alpha_{h} \exp \left\{\alpha \sum_{h=1}^{L} W_{h}\left(\bar{x}_{h}^{\prime} \frac{\bar{z}}{\sum_{h=1}^{L} W_{h} \bar{z}_{h}^{\prime}}-\bar{x}_{h}\right) / \sum_{h=1}^{L} W_{h}\left(\bar{x}_{h}^{\prime} \frac{\bar{z}}{\sum_{h=1}^{L} W_{h} \bar{z}_{h}^{\prime}}+(a-1) \bar{x}_{h}\right)\right]\right]
$$

where $\lambda, \alpha$ and $a$ are contants and minimum MSE of the above estimator is

$$
\operatorname{MSE}\left(\hat{\bar{Y}}_{S}\right)_{\min }=\bar{Y}^{2}\left[1+\left\{1+V_{200}-\frac{\left(V_{110}^{\prime}-V_{110}-V_{101}^{\prime}\right)^{2}}{V_{020}-V_{020}^{\prime}+V_{002}^{\prime}}\right\}^{-1}\right]
$$

\section{PROPOSED ESTIMATOR}

Motivated by Sanaulla et. al. (2014), the following class of exponential estimator for estimation of population mean under double stratified random sampling has been proposed,

$$
\hat{\bar{Y}}_{R P}^{s}=\left[\sum_{h=1}^{L} W_{h} \bar{y}_{h}+k\left\{W_{h}\left(\bar{x}_{h}^{\prime}-\bar{x}_{h}\right)\right\}\right]\left[\alpha H_{1}+(1-\alpha) H_{2}\right]
$$

where

$$
H_{1}=\exp \left[\left\{\sum_{h=1}^{L} W_{h}\left(\bar{x}_{h}^{\prime} \frac{\bar{z}}{\sum_{h=1}^{L} W_{h} \bar{z}_{h}^{\prime}}-\bar{x}_{h}\right)\right\} /\left\{\sum_{h=1}^{L} W_{h}\left(\bar{x}_{h}^{\prime} \frac{\bar{z}}{\sum_{h=1}^{L} W_{h} \bar{z}_{h}^{\prime}}+\bar{x}_{h}\right)\right]\right]
$$

and

$$
H_{2}=\exp \left[\left\{\sum_{h=1}^{L} W_{h}\left(\bar{x}_{h}-\bar{x}_{h}^{\prime} \frac{\bar{z}}{\sum_{h=1}^{L} W_{h} \bar{z}_{h}^{\prime}}\right)\right\} /\left\{\sum_{h=1}^{L} W_{h}\left(\bar{x}_{h}+\bar{x}_{h}^{\prime} \frac{\bar{z}}{\sum_{h=1}^{L} W_{h} \bar{z}_{h}^{\prime}}\right)\right]\right]
$$

Further simplifying using (1), (2) and (3), we have 


$$
\begin{aligned}
\left(\hat{\bar{Y}}_{R P}^{s}-Y\right)^{2}=\bar{Y}^{2} & {\left[\alpha^{2}\left(e_{1}^{\prime}-e_{1}-e_{2}^{\prime}\right)^{2}+\frac{1}{4}\left(e_{1}^{\prime}-e_{1}-e_{2}^{\prime}\right)^{2}+e_{0}^{2}-\alpha\left(e_{1}^{\prime}-e_{1}-e_{2}^{\prime}\right)^{2}-e_{0}\left(e_{1}^{\prime}-e_{1}-e_{2}^{\prime}\right)\right.} \\
& \left.+2 \alpha e_{0}\left(e_{1}^{\prime}-e_{1}-e_{2}^{\prime}\right)+k^{2} \bar{X}^{2}\left(e_{1}^{\prime}-e_{1}\right)^{2}+(2 \alpha-1) k \bar{X} \bar{Y}\left(e_{1}^{\prime}-e_{1}\right)\left(e_{1}^{\prime}-e_{1}-e_{2}^{\prime}\right)+2 k \bar{X} \bar{Y} e_{0}\left(e_{1}^{\prime}-e_{1}\right)\right]
\end{aligned}
$$

Taking expectation, we have

$$
\begin{aligned}
\operatorname{MSE}\left(\hat{\bar{Y}}_{R P}^{s}\right)= & \bar{Y}^{2}\left[\alpha^{2}\left(V_{020}-V_{020}^{\prime}+V_{002}^{\prime}\right)+\frac{1}{4}\left(V_{020}-V_{020}^{\prime}+V_{002}^{\prime}\right)+V_{200}-\alpha\left(V_{020}-V_{020}^{\prime}+V_{002}^{\prime}\right)-\left(V_{110}^{\prime}-V_{110}+V_{101}^{\prime}\right)\right. \\
& \left.\left.+2 \alpha\left(V_{110}^{\prime}-V_{110}+V_{101}^{\prime}\right)\right]+k^{2} \bar{X}^{2}\left(V_{020}-V_{020}^{\prime}\right)+(2 \alpha-1) k \bar{X} \bar{Y}\left(V_{020}-V_{020}^{\prime}\right)+2 k \bar{X} \bar{Y} e_{0}\left(V_{110}^{\prime}-V_{110}\right)\right]
\end{aligned}
$$

Finding optimum value of $k$ and $\alpha$ from (11) we get

$$
\begin{aligned}
& k_{(o p t)}=\frac{\bar{Y}}{\bar{X}}\left[\frac{V_{101}^{\prime}}{V_{002}^{\prime}}-\frac{\left(V_{110}^{\prime}-V_{110}\right)}{\left(V_{020}-V_{020}^{\prime}\right)}\right] \\
& \alpha_{(o p t)}=\frac{1}{2}+\frac{V_{101}^{\prime}}{V_{200}^{\prime}}
\end{aligned}
$$

By substituting the optimum value of $k_{\text {opt }}$ and $\alpha_{\text {opt }}$ the minimum MSE is,

$$
\operatorname{MSE}\left(\hat{\bar{Y}}_{R P}^{s}\right)_{\min }=\bar{Y}^{2}\left[V_{200}-\frac{V_{101}^{\prime 2}}{V_{002}^{\prime}}-\frac{\left(V_{110}^{\prime}-V_{110}\right)^{2}}{V_{020}-V_{020}^{\prime}}\right]
$$

Putting $k=0$ and finding optimum value of $\alpha$, we get

$$
\alpha_{o p t}=\frac{1}{2}-\left[\frac{V_{110}^{\prime}-V_{110}-V_{101}^{\prime}}{V_{020}-V_{020}^{\prime}+V_{002}^{\prime}}\right]
$$

and using (19) in (11), we have

$$
\operatorname{MSE}\left(\hat{\bar{Y}}_{R P}^{s}\right)_{\min }=\bar{Y}^{2}\left[V_{200}-\frac{\left(V_{110}^{\prime}-V_{110}-V_{101}^{\prime}\right)^{2}}{V_{020}-V_{020}^{\prime}+V_{002}^{\prime}}\right]
$$

\section{EFFICIENCY COMPARISONS}

For carrying out efficiency comparison, the proposed estimator is compared with the above discussed estimators and modified form of Hansen-Hurwitz unbiased estimator.

$$
\operatorname{MSE}\left(\hat{\bar{Y}}_{R P}^{s}\right)<\operatorname{Var}\left(\bar{y}_{s t}\right) \text { if }
$$




$$
\begin{aligned}
& \frac{V_{101}^{\prime 2}}{V_{002}^{\prime}}+\frac{\left(V_{110}^{\prime}-V_{110}\right)^{2}}{V_{020}-V_{020}^{\prime}} \geq 0 \\
& \operatorname{MSE}\left(\hat{\bar{Y}}_{R P}^{s}\right)<\operatorname{MSE}\left(\bar{y}_{R d}\right) \text { if } \\
& \frac{V_{101}^{\prime 2}}{V_{002}^{\prime}}+\frac{\left(V_{020}-V_{020}^{\prime}+V_{110}^{\prime}-V_{110}\right)^{2}}{V_{020}-V_{020}^{\prime}} \\
& \operatorname{MSE}\left(\hat{\bar{Y}}_{R P}^{s}\right)<\operatorname{MSE}\left(\bar{y}_{P d}\right) \text { if } \\
& \frac{V_{101}^{\prime 2}}{V_{002}^{\prime}}+\frac{\left(V_{020}-V_{020}^{\prime}-V_{110}^{\prime}+V_{110}\right)^{2}}{V_{020}-V_{020}^{\prime}} \\
& \operatorname{MSE}\left(\hat{\bar{Y}}_{R P}^{s}\right)<\operatorname{MSE}\left(y_{\text {reg }}^{R}\right) \text { if } \\
& \frac{V_{101}^{\prime 2}}{V_{002}^{\prime}}-2 V_{101}^{\prime}+V_{002}^{\prime} \geq 0 \\
& \operatorname{MSE}\left(\hat{\bar{Y}}_{R P}^{s}\right)<\operatorname{MSE}\left(y_{\text {reg }}^{P}\right) \text { if } \\
& \frac{V_{101}^{\prime 2}}{V_{002}^{\prime}}+2 V_{101}^{\prime}+V_{002}^{\prime} \geq 0 \\
& \operatorname{MSE}\left(\hat{\bar{Y}}_{R P}^{s}\right)<\operatorname{MSE}\left(\hat{\bar{Y}}_{S}\right)_{\min } \text { if } \\
& \frac{\left[V_{110}^{\prime}\left(V_{020}-V_{020}^{\prime}\right)+V_{020}^{\prime}\left(V_{110}^{\prime}-V_{110}\right)\right]^{2}}{V_{002}^{\prime}\left(V_{020}-V_{020}^{\prime}\right)\left(V_{110}^{\prime}-V_{110}\right)} \geq 0
\end{aligned}
$$

\section{EMPIRICAL STUDY}

To carry out the numerical illustrations some industrial datasets are used to check the usefulness of the proposed estimators of $\bar{Y}$,

Dataset-I: "Source: (Murthy, 1967) Y: Production (Output), X: No. of workers, Z: Fixed Capital"

Dataset-II: "Source: (Sardanal et al., 1992) Y: 1983 Workers (in millions), X: 1980 Workers (in millions), Z: 1982 Gross National Product (in tens of millions of U.S. dollars)"

Dataset-III: "Source: (Gujarati, 2003) Y: Average miles per gallon, X: Engine horsepower, Z: Top speed, miles per hour" 
Table 1: Dataset-I

\begin{tabular}{c|c|c|c|c|c|c|c|c|c|c|c|c}
\hline Strata & $N_{h}$ & $n_{h}$ & $n_{h}^{\prime}$ & $S_{y h}$ & $S_{x h}$ & $S_{z h}$ & $\bar{Y}_{h}$ & $\bar{X}_{h}$ & $\bar{Z}_{h}$ & $\rho_{x y h}$ & $\rho_{x z h}$ & $\rho_{y z h}$ \\
\hline 1 & 19 & 5 & 11 & 757.08 & 11.18 & 109.45 & 65.16 & 2669.25 & 349.68 & 0.81 & 0.90 & 0.93 \\
\hline 2 & 32 & 8 & 17 & 669.11 & 44.35 & 109.22 & 140.00 & 4657.62 & 706.60 & 0.88 & 0.84 & 0.92 \\
\hline 3 & 14 & 3 & 8 & 417.00 & 81.11 & 277.18 & 403.21 & 6537.21 & 1539.60 & 0.92 & 0.93 & 0.98 \\
\hline 4 & 15 & 4 & 9 & 645.69 & 171.44 & 370.96 & 763.20 & 7843.67 & 2620.53 & 0.97 & 0.94 & 0.96 \\
\hline
\end{tabular}

Table 2: Dataset-II

\begin{tabular}{c|c|c|c|c|c|c|c|c|c|c|c|c}
\hline Strata & $N_{h}$ & $n_{h}$ & $n_{h}^{\prime}$ & $S_{y h}$ & $S_{x h}$ & $S_{z h}$ & $\bar{Y}_{h}$ & $\bar{X}_{h}$ & $\bar{Z}_{h}$ & $\rho_{x y h}$ & $\rho_{x z h}$ & $\rho_{y z h}$ \\
\hline 1 & 38 & 16 & 27 & 16.46 & 14.91 & 1915.17 & 11.88 & 13.03 & 1029.16 & 0.99 & 0.74 & 0.74 \\
\hline 2 & 14 & 6 & 10 & 60.23 & 58.48 & 78097.92 & 26.18 & 27.35 & 25671.57 & 0.99 & 0.97 & 0.96 \\
\hline 3 & 11 & 4 & 8 & 34.89 & 32.66 & 7588.80 & 21.88 & 23.13 & 5028.82 & 0.99 & 0.97 & 0.97 \\
\hline 4 & 33 & 14 & 23 & 209.08 & 200.07 & 20672.75 & 75.24 & 79.65 & 7533.94 & 0.99 & 0.30 & 0.29 \\
\hline 5 & 24 & 10 & 17 & 18.80 & 18.69 & 19782.83 & 20.09 & 20.28 & 16314.42 & 0.99 & 0.90 & 0.90 \\
\hline
\end{tabular}

Table 3: Dataset-III

\begin{tabular}{c|c|c|c|c|c|c|c|c|c|c|c|c}
\hline Strata & $N_{h}$ & $n_{h}$ & $n_{h}^{\prime}$ & $S_{y h}$ & $S_{x h}$ & $S_{z h}$ & $\bar{Y}_{h}$ & $\bar{X}_{h}$ & $\bar{Z}_{h}$ & $\rho_{x y h}$ & $\rho_{x z h}$ & $\rho_{y z h}$ \\
\hline 1 & 21 & 06 & 15 & 12.14 & 76.71 & 19.48 & 37.55 & 116.57 & 114.14 & -0.7914 & 0.9894 & -0.7781 \\
\hline 2 & 34 & 04 & 17 & 8.34 & 31.94 & 07.10 & 37.25 & 93.00 & 106.50 & -0.8339 & 0.8820 & -0.6651 \\
\hline 3 & 26 & 02 & 08 & 5.47 & 49.55 & 13.21 & 26.39 & 26.39 & 118.88 & -0.7696 & 0.9669 & -0.5935 \\
\hline
\end{tabular}

In Table 5 the Percentage Relative Efficiencies (PREs) of different suggested estimators of $\bar{Y}$ with respect to usual estimator $\bar{y}_{s t}$ using the formula given below are tabulated.

$$
\operatorname{PRE}\left(*, \bar{y}_{s t}\right)=\frac{V\left(\bar{y}_{s t}\right)}{V(*)}
$$

where * stands for $\operatorname{MSE}\left(\bar{y}_{R d}\right), \operatorname{MSE}\left(\bar{y}_{P d}\right), \operatorname{MSE}\left(y_{\text {reg }}^{R}\right), \operatorname{MSE}\left(y_{\text {reg }}^{P}\right), \operatorname{MSE}\left(\hat{\bar{Y}}_{S}\right)$ and $\operatorname{MSE}\left(\hat{\bar{Y}}_{R P}^{s}\right)$ 
Table 5: PREs of the estimators of $\bar{Y}$

\begin{tabular}{c|c|c|c}
\hline \multirow{2}{*}{ Estimators } & \multicolumn{3}{|c}{ PREs } \\
\cline { 2 - 4 } & Dataset-I & Dataset-II & Dataset-III \\
\hline $\bar{y}_{s t}$ & 100.00 & 100.00 & 100.00 \\
\hline $\bar{y}_{R d}$ & 13.00 & 101.00 & $*$ \\
\hline $\bar{y}_{P d}$ & $*$ & $*$ & 29.00 \\
\hline$y_{\text {reg }}^{R}$ & 18.73 & 151.97 & 52.60 \\
\hline$y_{\text {reg }}^{P}$ & $*$ & $*$ & 193.58 \\
\hline$\hat{\bar{Y}}_{S}$ & 136.05 & 152.32 & 208.30 \\
\hline$\hat{\bar{Y}}_{R P}^{s}$ & 143.00 & 203.90 & \\
\hline
\end{tabular}

* Data not applicable for this estimator

\section{CONCLUSION}

In Table-4, it can be noticed with the help of percent relative efficiencies (PREs) that the proposed class of estimators - clearly - outdo the usual unbiased estimator in double stratified sampling $\bar{y}_{s t}$, ratio and product estimators $\bar{y}_{R d}$ and $\bar{y}_{P d}$ respectively. Also it outperform Mohanty (1967) regression-cum-ratio and regression-cum-product estimators $y_{\text {reg }}^{R}$ and $y_{\text {reg }}^{P}$ respectively. It is also superior to Sanaullah et al. (2014) estimators $\hat{\bar{Y}}_{S}$ for the population mean $\bar{Y}$. So, in practice the use of the suggested class of estimators could be preferred instead of existing methods to computing statistical inference for industrial data and others for higher accuracy in the estimates.

\section{ACKNOWLEDGMENTS}

The authors are thankful to the editor-in-chief Professor Vladimir Kvasnicka and learned reviewers for their valuable comments towards the improvements of the manuscript. 


\section{REFERENCES}

Bahl, S., Tuteja, R.K., 1991. Ratio and product type exponential estimators, Journal of Information and Optimization Sciences, 12, 1, 159-164.

Chand, L., 1975. Some ratio-type estimators based on two or more auxiliary variates, Unpublished Ph.D. dissertation, Iowa State University, Ames, Iowa.

Cingi, H., Kadilar, C., Kocberber, G., 2007. Investigation of first and secondary education opportunities in turkey and improving solution proposals to specified knowledge, Tubitak, Project No: 106K077.

Gujarati, D.N., 2003. Basic econometrics, McGraw Hill United States Military Academy, West Point.

Kadilar, C., Cingi, H., 2003. Ratio estimators in stratified random sampling, Biometrical Journal, 45, 2, 218-225.

Kadilar, C., Cingi, H., 2005. A new ratio estimator in stratified random sampling, Communications in Statistics - Theory and Methods, 34, 3, 597-602.

Koyuncu, N., Kadilar, C., 2009. Ratio and product estimators in stratified random sampling, Journal of Statistical Planning and Inference, 139, 8, 2552-2558.

Mohanty, S., 1967. Combination of regression and ratio estimate, Journal of Indian Statistical Association, 5, 16-19.

Murthy, M. N., 1967. Sampling theory and methods, Statistical Publishing Society, Calcutta, India.

Samiuddin, M., Hanif, M., 2007. Estimation of population mean in single and two phase sampling with or without additional information, Pakistan Journal of Statistics-All Series, 23, 2, 99.

Sanaullah, A., Ali, H. A., ul Amin, M. N., Hanif, M., 2014. Generalized exponential chain ratio estimators under stratified two-phase random sampling, Applied Mathematics and Computation, 226, 541-547.

Sarndal, C.E., Swensson, B., Wretman, J., 1992. Model assisted survey sampling, Springer-Verlag, New York.

Shabbir, J., Gupta, S., 2005. Improved ratio estimators in stratified sampling, American Journal of Mathematical and Management Sciences, 25, 3-4, 293-311.

Singh, H. P., Vishwakarma, G. K., 2007. Modified exponential ratio and product estimators for finite population mean in double sampling, Austrian Journal of Statistics, 36, 3, 217-225.

Singh, R., Chauhan, P., Swan, N., Smarandache, F., 2011. Improved exponential estimator for population variance using two auxiliary variates, Italian Journal of Pure and Applied Mathematics, 28, 101-108.

Upadhyaya, L. N., Singh, H. P., 1999. An estimator for population variance that utilizes the kurtosis of an auxiliary variate in sample surveys, Vikram Mathematical Journal, 19, 1, 14-17.

Vishwakarma, G. K., Singh, H. P., 2015. Class of chain ratio-type estimators of finite population mean in stratified two-stage sampling, Proceedings of the National Academy of Sciences, India Section A: Physical Sciences, 85, 1, 99-115. 
Sayed Mohammed Zeeshan

School of Advanced Sciences \& Languages

Vellore Institute of Technology, Bhopal-466114, India

E-mail: zeeshan008x52@gmail.com

Gajendra K. Vishwakarma

Department of Mathematics \& Computing,

Indian Institute of Technology Dhanbad, Dhanbad-826004, India

E-mail: vishwagk@rediffmail.com 